\title{
Basal serum follicle-stimulating hormone and anti- mullerian hormone are the predictors of poor ovarian response among fertile and infertile women below the age of 40 years
}

\section{Haroon Latif Khan}

Lahore Institute of Fertility and Endocrinology, Hameed Latif Hospital

Shahzad Bhatti ( $\sim$ drshahzadbhatti@yahoo.com )

Lahore Institute of Fertility and Endocrinology, Hameed Latif Hospital Imrana

Lahore Institute of Fertility and Endocrinology, Hameed Latif Hospital Humera

Lahore Institute of Fertility and Endocrinology, Hameed Latif Hospital Samina Shuail

Lahore Institute of Fertility and Endocrinology, Hameed Latif Hospital

\section{Research Article}

Keywords: Female infertility, FSH, LH, poor ovarian reserves

Posted Date: March 3rd, 2021

DOI: https://doi.org/10.21203/rs.3.rs-294060/v1

License: (c) (1) This work is licensed under a Creative Commons Attribution 4.0 International License. Read Full License 


\section{Abstract}

Infertility is defined as the inability to conceive despite having frequent, unprotected sex for at least one year. The failure to have children affects men and women across the globe. Infertility can lead to distress and depression [1]. The clinical definition of infertility used by the World Health Organization (WHO) is "a disease of the reproductive system defined by the failure to achieve a clinical pregnancy after 12 months or more of regular unprotected sexual intercourse" [2], while the WHO's epidemiologic definition is "women of reproductive age at risk of becoming pregnant who report unsuccessfully trying for a pregnancy for more than two years" [3]. Clinical definitions are designed for early detection and treatment of infertility [2][4]. A progressive decline in fecundity occurs in both males and females with advancing age. Generally, male factors contribute to one-third of infertility cases, the other one-third occurs due to female reproductive deficiencies, while in remaining cases, both male and female causes equally contribute towards infertility. In some cases, infertility is iatrogenic in nature as both male and female profile comes out to be normal and termed as unexplained infertility.

\section{Introduction}

Infertility is defined as the inability to conceive despite having frequent, unprotected sex for at least one year. The failure to have children affects men and women across the globe. Infertility can lead to distress and depression [1]. The clinical definition of infertility used by the World Health Organization (WHO) is "a disease of the reproductive system defined by the failure to achieve a clinical pregnancy after 12 months or more of regular unprotected sexual intercourse" [2], while the WHO's epidemiologic definition is "women of reproductive age at risk of becoming pregnant who report unsuccessfully trying for a pregnancy for more than two years" [3]. Clinical definitions are designed for early detection and treatment of infertility [2][4]. A progressive decline in fecundity occurs in both males and females with advancing age. Generally, male factors contribute to one-third of infertility cases, the other one-third occurs due to female reproductive deficiencies, while in remaining cases, both male and female causes equally contribute towards infertility. In some cases, infertility is iatrogenic in nature as both male and female profile comes out to be normal and termed as unexplained infertility.

Although Pakistan is among the world's currently most populous countries and has a population growth rate of around $2 \%$, it also has a high infertility rate (21.9\%); $3.5 \%$ primary, and $18.4 \%$ secondary ${ }^{[5]}$. This shows that one in five couples in our country is directly related to this problem. Among various risk factors leading to female infertility, advancing age is imperative. A gradual decline in women's reproductive capabilities begins in their mid-30s, and a steep decline occurs rapidly after age 37 , and by age 40 , fertility gets reduced by $50 \%$. Infertility in old age may be due to poor ovarian reserve that is a decline in the number and quality of eggs or various health problems that affect fertility. With advancing 
age, chances of conception gradually decline. Also, a risk of miscarriages, genetic abnormalities in newborns, and complications in pregnancy and childbirth increases.

Female fertility is directly related to ovarian reserve, which is the ovary's capacity to provide egg cells capable of fertilization, resulting in healthy and successful pregnancy [6]. It is at its peak in young women of age below 30s, but it declines and eventually leads to menopause with advancing age. Some biochemical parameters such as FSH and $\mathrm{AMH}$ are essential predictors of current ovarian reserve. In the ovaries, $\mathrm{AMH}$ is secreted in early-stage follicles by the granulosa cells and inhibits the FSH stimulated the growth of follicles. FSH and AMH's respective role in defining functional ovarian reserve is to better elucidate the association between $\mathrm{FSH}, \mathrm{AMH}$, and oocyte quality; we decided to explore mathematical models of functional ovarian reserve.

Serum FSH and AMH levels are directly correlated to each other and with age, which affects the pregnancy outcomes. As FSH increases with age, AMH levels decline. AMH is the serum marker whose fluctuation determines the current fertility status of the female. As it decreases at an early age, even if FSH is within the normal range, it shows poor egg quality on ovum pick up, interfering with fertilization and desired pregnancy outcomes.

$\mathrm{AMH}$ level analyses have been done frequently at various points during the menstrual cycle, suggesting a complete absence of fluctuation. It remains stable throughout the cycle and after administration of oral or vaginal administration of synthetic sex steroids. $\mathrm{AMH}$, independent of age, has some association with predicting live birth after assisted conception and may help counseling couple before undergoing fertility treatment. The routine uses of AMH levels before IVF/ICSI in patients considered at high risk for cycle cancellation significantly reduced the incidence of cycle cancellation as compared with a controlled group with no AMH assays.

Circulating AMH can predict excessive and poor response to stimulation with exogenous gonadotrophins; overall, this biomarker is supervision to basal FSH and AFC, and has the potential to be incorporated into workup protocols to predict patient's ovarian response.

AMH cannot be detected in the female until puberty and reaches its maximum level at 24.5 years of age. The age-related decrease in female fertility can be most likely attributable to deterioration in oocytes' quantity and quality. Because of diminished ovarian reserve, the woman's ability to conceive naturally and with IVF will limit after 40. Although assumptions indicate that the relationship between age and ovarian reserve is highly variable and potential different validity of ovarian reserve markers in women in different age groups remains to be demonstrated. Few studies assess these markers' predictive value as $\mathrm{AMH}, \mathrm{FSH}$, and AFC may have different accuracy in different ages.

Prediction of poor response is vital for the counseling and management of these women in clinical practice based on a low AFC or reduced AMH levels. Currently, AMH should be considered the more reliable of the ovarian reserve assessment test than $\mathrm{FSH}$; there is a strong positive correlation between 
serum AMH level and AFCA. The use of AMH combined with AFC may improve ovarian reserve evaluation.

In the ovary, AMH inhibits initial primordial follicle recruitment and decreases the sensitivity of pre-antral and small antral follicles to FSH.

The current study aimed to examine $\mathrm{AMH}, \mathrm{FSH}$, and AFC's predictive value to predict clinical/chemical pregnancy rate, live birth, and ovarian response to controlled ovarian hyperstimulation in an IVF cycle in a population with different age groups.

\section{Materials \& Methods}

\section{Study design:}

It was a retrospective study on couples coming to treat subfertility in the Lahore institute of fertility and endocrinology (LIFE). This study was approved by an institutional ethical review committee of Hameed Latif hospital.

Physical parameters: They were divided into three groups depending on age, as age less than 35 years, between 35- 40 and above 40. Data were collected from July 2017 onwards from 311 infertile women who underwent assisted fertilization at LIFE.

Inclusion criteria: The women included in this study were aged between 25-36 years, with unexplained factors as a cause of subfertility.

Exclusion criteria: Women with severe PCOS (Polycystic Ovarian Syndrome) and endometriosis will be excluded from this study. Also, women with any pelvic pathology were excluded. Furthermore, couples with azoospermia and severe oligoasthenospermia were excluded.

Preliminary assessment: Preliminary examination was done, including hormonal profile such as FSH, prolactin, and E2 (Estradiol) on the 2nd day of the menstrual cycle. Moreover, thyroid profile T3, T4, TSH, and screening tests including hepatitis profile, $C B C$, and blood sugar were done. A transvaginal scan (EUB 5500 Hitachi) to exclude any pelvic pathology was done. Moreover, antral follicle count and uterine status, endometrial thickness was also measured.

Protocol in ART: Pituitary down-regulation was started during the mid-luteal phase with subcutaneous decapeptide (triptorelin acetate) $0.1 \mathrm{mg}$ daily. The stimulation drugs were administered on the $2^{\text {nd }}-3^{\text {rd }}$ Days of the cycle in required dosages, and regular monitoring was carried out by ultrasound and serial Estradiol levels. The stimulating drugs were either human menopausal gonadotrophins or follicular stimulating hormones. Recombinant HCG (IVF-C Galaxy) was given at a specific follicular size (18$20 \mathrm{~mm}$ ), and egg retrieval in dosage 5000 or $10000 \mathrm{IU}$ was performed 36 hours later; Embryo transfer was done 3-5 days later. 
Data Analysis: SPSS 25.0 will be used for data analysis; descriptive analysis will be done, such as frequencies and percentages of categorical variables and mean standard deviation, and variance for numerical variables. Chi-square test and Kruskal Wallis test will be used to check the association between variables within different age groups. The level of significance will be 0.05 or $5 \%$.

\section{Results}

In total, 311 IVF patients were included in this study. The average age was $32.15 \pm 4.38$ years. The average BMI was $27.94 \pm 4.65 \mathrm{~kg} / \mathrm{m}^{2}$. The average $\mathrm{AMH}$ and $\mathrm{FSH}$ was $3.33 \pm 2.08,8.16 \pm 4.64$, respectively. The average AFC was $6.86 \pm 2.15$.

The patients were divided by age into three groups: group A ( $\leq 25 \mathrm{yrs})$ consists of $6.1 \%$ (19) patients, in group B (26-35yrs) were 73.3\% (228) and in group C (> 35yrs) were 20.6\% (64) patients. All these groups have unexplained subfertility. Primary infertility was present in 159 patients, and secondary was in 152 . The mean infertility duration in group $A$ was $3 \pm 1.64$, in group $B 4 \pm 2.51$, and in group, $C$ was $7 \pm 4.63$. Significant association was present between-group AC ( $p$-value $=0.00)$ and group BC ( $p$-value 0.00$)$

\section{Baseline endocrine parameters}

The median $(95 \% \mathrm{Cl})$ of FSH was 7.20 (4.47-9.83) in group A, 7.48 (6.55-9.05) in group B and 7.30 (4.71$10.87)$ in group $C$. In group $A$, the median of $A M H$ was 3.68 (0.84-5.83), in group $B$ it was 3.13 (2.82-5.37) and $1.93(0.26-4.10)$ in group $C$.

The median of TSH was 1.53 (0.36-2.68) in group A, 1.59 (1.23-2.05) in group B and 2.38 (0.34-4.73) in group C. Prolactin was 18.50 (5.65-28.08), 23.70 (12.04-17.53) and 14.40 (5.84-25.23) in group A, B and C respectively. In group A, B and C, the median of LH was 6.61 (3.26-11.70), 3.90 (3.70-5.18) and 4.54 (1.559.15). Similarly median of Estradiol (E2) was 54.55 (27.46-80.14), 47.05 (35.72-106.50) and 46.60 (22.2483.68) in group $A, B$ and $C$ respectively. All endocrine hormones were insignificant except $A M H$ ( $p$-value $=0.00)$.

\section{In ultrasonography parameters}

Antral follicular count (AFC), in group A the median (95\% Cl) was 8 (7.45-9.04), ), in group B 7 (6.08-7.91) and in group $C$ 8(5.36-11.03). Endometrium thickness varies with age as in group $A$, the median $(95 \% \mathrm{Cl})$ was 5.49 (2.39-9.03), 7 (5.90-7.88) in group C and 7.50 (3.87- 9.87) in group C.

As shown in table 2, the mean number of days of stimulation was 12 in Group A, B and 13 in group C. Number of days of stimulation was not significantly different in various age groups. ( $P$-value $=0.29$ )

As for starting dose of stimulatory drugs, the median was 1725IU in group A, 2200IU in group B, and $2475 \mathrm{IU}$ in group C. Group $\mathrm{C}$ has a higher dose of a stimulatory drug than to group A and B. According to the $p$-value (0.01), there was a statistically significant relationship between groups $A$ and $C$. And there 
was no significant difference between groups $A$ and $B$, and no significance was present between groups $B$ and $C$ ( $p$-value $=0.24,0.08$ ) respectively.

The endocrine parameters on decision day in groups $A, B$, and $C$ were not significantly different. Regarding endometrial thickness, the median was 10 in all groups. $(p=0.85)$ The median of follicles in groups $A$ and $B$ was 20 and 17 in group $C$. There was a significant relationship between follicles and age. ( $P$-value $=0.02$ in group $B$ and $C)$. (Table 2 )

There was a negative correlation between the number of follicles $(p=0.00)$, the number of eggs retrieved $(p=0.05)$, the number of fertilized embryos $(p=0.15)$, and several cleaved embryos $(p=0.03)$ concerning age, and also a negative correlation was present between a number of transferred embryos and age and statistically insignificant. (Table 3 )

$26 \%$ (81) were positive, and $66.2 \%$ (206) were negative and the remaining had no embryo transfer due to some issues. (Table 4)

\section{Discussion}

This study was conducted at Lahore Institute of fertility and endocrinology (LIFE); we compared the incidence of infertility in different age groups and their possible association with hormone levels. Malefactors, tubal, endometriosis, and PCOS were not included in our study. There was a total of 311 women who presented with unexplained infertility complaints, so we divided them into three age groups and carried out tests including different hormone levels and transvaginal ultrasonography to assess antral follicle count that could have possibly explained the cause of infertility in these patients.

In our study, we compare primary and secondary infertility with age showed no significant difference, whereas previous researches show, the rate of infertility should have been enhanced with advancing age.

Similarly, infertility duration between these groups showed no remarkable difference. A study carried out in Iraq showed that out of 250 couples, $193(77.2 \%)$ had primary while $57(22.8 \%)$ had secondary infertility. ${ }^{[7]}$ In another study, primary infertility was $8 \%$, whereas secondary infertility was $4.6 \%$. ${ }^{[8]}$ In our results, the rate of primary infertility is higher than secondary. Infertility affected both partners; it may be primary or secondary. The inability to having babies or sex of the baby has a negative impact on their relationship. [9]

Age, basal FSH, AFC, and inhibin B are the most critical components for assessing ovarian reserve. To further explain the likely cause of unexplained infertility in these women, we conducted baseline endocrine parameters as shown in table 1. FSH levels in groups A and B were almost the same; group $C$ showed a slight increase but was insignificant. Generally, it is accepted that FSH is more effective than age in predicting the IVF/ICSI ovarian response and cycle cancellation rate, whereas age is more effective in predicting the IVF pregnancy rate. ${ }^{[10]}$ Another study suggested that in the IVF procedure as well as the 
outcome, FSH level was a key indicator. [11]. The chance of conception is reduced in advanced age due to the decline of ovarian reserve.

Many years ago, physicians relied on $\mathrm{FSH}$ and other hormones rather than $\mathrm{AMH}$. $\mathrm{AMH}$ concentration is higher in the $36^{\text {th }}$ week of gestation, which is slightly decreased during puberty, and at the stage of menopause, it becomes undetectable. ${ }^{[12,13]}$ The normal range of $A M H$ is $1.0-4.0 \mathrm{ng} / \mathrm{ml}$; below $1.0 \mathrm{ng} / \mathrm{ml}$ is considered as a low level for fertile women. ${ }^{[14]}$ However, comparison of $\mathrm{AMH}$ levels with age showed some significant outcomes as group $C$ ( $>35$ age) had a value of 1.93 compared to group $A$ and $B$ with values 3.68 and 3.49, respectively. According to theory and other researches, an AMH value above 1 is still assumed to be enough for women to conceive, but in our studies, women showed infertility despite having $\mathrm{AMH}$ levels in the normal range. However, with age, a reduction in $\mathrm{AMH}$ levels is significant.

TSH levels showed little elevation with age but not significant. Similar outcomes analyzed for Prolactin levels. Luteinizing Hormone, Estrogen, and progesterone levels reduced with advancing age which is normal.

We also included ultrasonography parameters as shown in table 1 as ovarian reserves analysis was crucial in reaching a possible conclusion. Antral Follicle count in three age groups shown to have no significant difference, two groups of different ages (A and B) showed similar AFC but decreased in group $C$ by increase the age.

According to pervious study, with the advancing age antral follicle count (AFC) and AMH hormone decreased and FSH hormone increased. When we compare the AMH and FSH with the AFC, AMH was a more steadfast biomarker of ovarian reserve. ${ }^{[15]}$. Our results are similar to this study.

Same results were seen while comparing the endometrial thickness in women of different ages which came out to be non-significant.

With the decline of age high dose of recombinant FSH show better results. A study recommended that use of FSH or GnRH antagonist protocols with higher dosages may decrease the cancellation rates. ${ }^{[16,17]}$

In this study different categories of age have negatively correlated with outcome parameters. Number of follicles, eggs retrieved, embryo cleavage shows significant correlation while fertilization rate and number of ETs were insignificant. Some theories explain declining oocyte quality with age. ${ }^{[18]}$ Other studies recommended that when the women cross 40 years the age, the number of oocytes were lesser and their quality was poor, affecting the fertilization rate, implantation rate, and IVF outcomes. ${ }^{[19,20]}$

$\mathrm{AMH}, \mathrm{AFC}$ and age predict possible cause of infertility in women and IVF outcomes significantly, but our study showed no significant difference in these parameters with age which could have been the possible reason of them to be infertile, that's why these infertile women are classified as having unexplained infertility, no single parameter indicated the possible cause of infertility in our group with advancing age. 


\section{References}

1. National, Regional, and Global Trends in Infertility Prevalence Since 1990: A Systematic Analysis of 277 Health Surveys

2. Zegers-Hochschild F, Adamson GD, de Mouzon J, Ishihara O, Mansour R, et al. (2009) The International Committee for Monitoring Assisted Reproductive Technology (ICMART) and the World Health Organization (WHO) revised glossary on ART terminology, 2009. Hum Reprod 24: 2683-2687 doi:10.1093/humrep/dep343. [PubMed]

3. World Health Organization (2006) Reproductive health indicators for global monitoring: guidelines for their generation, interpretation and analysis for global monitoring. Geneva: World Health Organization. $63 \mathrm{p}$.

4. Rowe PJ, Comhaire FH, Hargreave TB, Mellows HJ, Press CU (1993) WHO manual for the standardized investigation and diagnosis of the infertile couple. Cambridge: Cambridge University Press.

5. TAHIR, F., SHAHAB, M., AFZAL, M., SUBHAN, F., SULTAN, S., KAZI, B. M. AND DIL, A.S., 2004. Male reproductive health: An important segment towards improving reproductive health of a couple. In: Population research and policy development in Pakistan (eds. M.A. Chaudhry and M. Desousa), pp. 227248. Population Association of Pakistan, Islamabad, Pakistan.

6. https://en.wikipedia.org/wiki/Ovarian_reserve.

7. RAZZAK, A.H. AND WAIS, S.A., 2002. The infertile couple: a cohort study in Duhok, Iraq. East Mediterr. HIth. J., 8: 234-238

8. Safarinejad MR. Infertility among couples in a population-based study in Iran: prevalence and associated risk factors. Int J Androl 2008; 31: 303- 314

9. Psycho-Social Consequences of Secondary Infertility in Karachi. Neelofar Sami (Department of Community Health Sciences, Aga Khan University, Karachi. )

Tazeen Saeed Ali (Department of Community Health Sciences, Aga Khan University, Karachi.)

10. Creus M, Penarrubia J, Fabregues F, Vidal E, Carmona F, Casamitjana R, et al. Day 3 serum inhibin B and FSH and age as predictors of assisted reproduction treatment outcome. Hum Reprod. 2000;15:23416

11. Van Loendersloot LL, van Wely M, Limpens J, Bossuyt PM, Repping S, van der Veen F. Predictive factors in in vitro fertilization (IVF): a systematic review and meta-analysis. Hum Reprod Update. 2010;16:577-89.

12. de Vet A, Laven JS, de Jong FH, Themmen AP, Fauser BC. Antimullerian hormone serum levels: a putative marker for ovarian aging. Fertil Steril 2002;77:357-62. 
13. Lee MM, Donahoe PK, Hasegawa T, Silverman B, Crist GB, Best S, et al. Mullerian inhibiting substance in humans: normal levels from infancy to adulthood. J Clin Endocrinol Metab 1996;81:571-6.

14. https://extendfertility.com/facts-figures/fertility-statistics-by-age

15. Barbakadze L, Kristesashvili J, Khonelidze N, Tsagareishvili G. The Correlations of Anti-Mullerian Hormone, Follicle-Stimulating Hormone and Antral Follicle Count in Different Age Groups of Infertile Women. Int J Fertil Steril 2015; 8: 393-398.

16. Akman MA, Erden HF, Tosun SB, Bayazit N, Aksoy E, Bahceci M. Comparison of agonistic flare-upprotocol and antagonistic multiple dose protocol in ovarian stimulation of poor responders: results of a prospective randomized trial. Hum Reprod 2001;16:868-70.

17. Craft I, Gorgy A, Hill J, Menon D, Podsiadly B. Will GnRH antagonists provide new hope for patients considered "difficult responders" to GnRH agonist protocols? Hum Reprod 1999;14:2959-62.

18. Eichenlaub-Ritter U. Genetics of oocyte ageing. Maturitas 1998;30: 143-69.

19. Hull MGR, Fleming CF, Hughes AO, McDermott A. The age-related decline in female fecundity: A quantitative controlled study of implanting capacity and survival of individual embryos after in vitro fertilization. Fertil Steril 1996; 65:783-90.

20. Viardot-Foucault V, Tai BC, Oo SY, et al. Cumulative success rates after IVF treatment: longitudinal study from a single centre. Reproductive Endocrinology and ART: A celebratory congress. Canada, 2011.

\section{Tables}




\begin{tabular}{|c|c|c|c|c|}
\hline \multirow[t]{4}{*}{ Characteristics } & & & & \multirow[t]{2}{*}{ p-value } \\
\hline & \multicolumn{3}{|l|}{ AGE (years) } & \\
\hline & A & B & C & \\
\hline & $\leq 25$ & $26-35$ & $>35$ & \\
\hline Number of women [n (\%)] & $19(6.1)$ & $\begin{array}{l}228 \\
(73.3)\end{array}$ & $64(20.6)$ & \\
\hline \multicolumn{5}{|l|}{ Infertility type [n (\%)] } \\
\hline Primary infertility & $15(9.4)$ & $\begin{array}{l}110 \\
(69.1)\end{array}$ & $34(21.5)$ & \multirow[t]{2}{*}{0.07} \\
\hline Secondary infertility & $4(2.6)$ & $\begin{array}{l}118 \\
(77.6)\end{array}$ & $30(18.8)$ & \\
\hline $\begin{array}{l}\text { Infertility duration (years) } \\
\text { (mean } \pm S . D)\end{array}$ & $4 \pm 1.89$ & $6 \pm 3.92$ & $8 \pm 5.00$ & $A B(0.45), A C, B C(0.00)$ \\
\hline \multicolumn{5}{|l|}{$\begin{array}{l}\text { Base line endocrine } \\
\text { parameters [ median ( } 95 \% \\
\text { C.I)] }\end{array}$} \\
\hline $\mathrm{FSH}$ & $\begin{array}{l}7.61(6.84- \\
9.01)^{(6.5}\end{array}$ & $\begin{array}{l}7.24 \\
(7.53- \\
8.92)\end{array}$ & $\begin{array}{l}8.12 \text { (7.37- } \\
9.17)\end{array}$ & 0.69 \\
\hline $\mathrm{AMH}$ & $\begin{array}{l}3.68(0.84- \\
5.83)\end{array}$ & $\begin{array}{l}3.49 \\
(2.95- \\
5.15)\end{array}$ & $\begin{array}{l}1.93(1.16- \\
3.34)\end{array}$ & $\mathrm{AC}(0.32), \mathrm{BC}(0.00), \mathrm{AB}(1.0)$ \\
\hline TSH & $\begin{array}{l}1.53(0.36- \\
2.68)\end{array}$ & $\begin{array}{l}1.59 \\
(1.29- \\
2.01)\end{array}$ & $\begin{array}{l}2.38(0.97- \\
3.80)\end{array}$ & 0.31 \\
\hline Prolactin & $\begin{array}{l}18.50(5.65- \\
28.08)\end{array}$ & $\begin{array}{l}14.25 \\
(13.32- \\
18.76)\end{array}$ & $\begin{array}{l}17.80(10.42- \\
22.82)\end{array}$ & 0.36 \\
\hline LH & $\begin{array}{l}6.61(3.26- \\
11.79)\end{array}$ & $\begin{array}{l}4.20 \\
(3.91- \\
5.23)\end{array}$ & $\begin{array}{l}4.80(2.88- \\
7.52)\end{array}$ & 0.19 \\
\hline E2 & $\begin{array}{l}54.55(27.46- \\
80.14)\end{array}$ & $\begin{array}{l}45.02 \\
(37.40- \\
96.25)\end{array}$ & $\begin{array}{l}46.60(19.69- \\
102.68)\end{array}$ & 0.83 \\
\hline \multicolumn{5}{|l|}{$\begin{array}{l}\text { Ultrasonography } \\
\text { parameters [ median (95\% } \\
\text { C.I)] }\end{array}$} \\
\hline Antral follicle count (AFC) & $\begin{array}{l}7(5.91- \\
8.61)\end{array}$ & $\begin{array}{l}7(6.63- \\
7.18)\end{array}$ & $6(6.10-7.12)$ & 0.41 \\
\hline Endometrial thickness & $5.7(4.88-$ & $6(5.73-$ & $5.4(5.02-$ & 0.62 \\
\hline
\end{tabular}




\section{Basal serum follicle stimulating hormone and anti mullerian hormone}

Table 1: Subjects clinical characteristics. FSH, follicle stimulating hormone; $\mathrm{AMH}$, anti mullerian hormone; TSH, thyroid stimulating hormone; E2, estradiol; AFC, antral follicle count . For the kruskal wallis test, chi square test for type of subfertility.

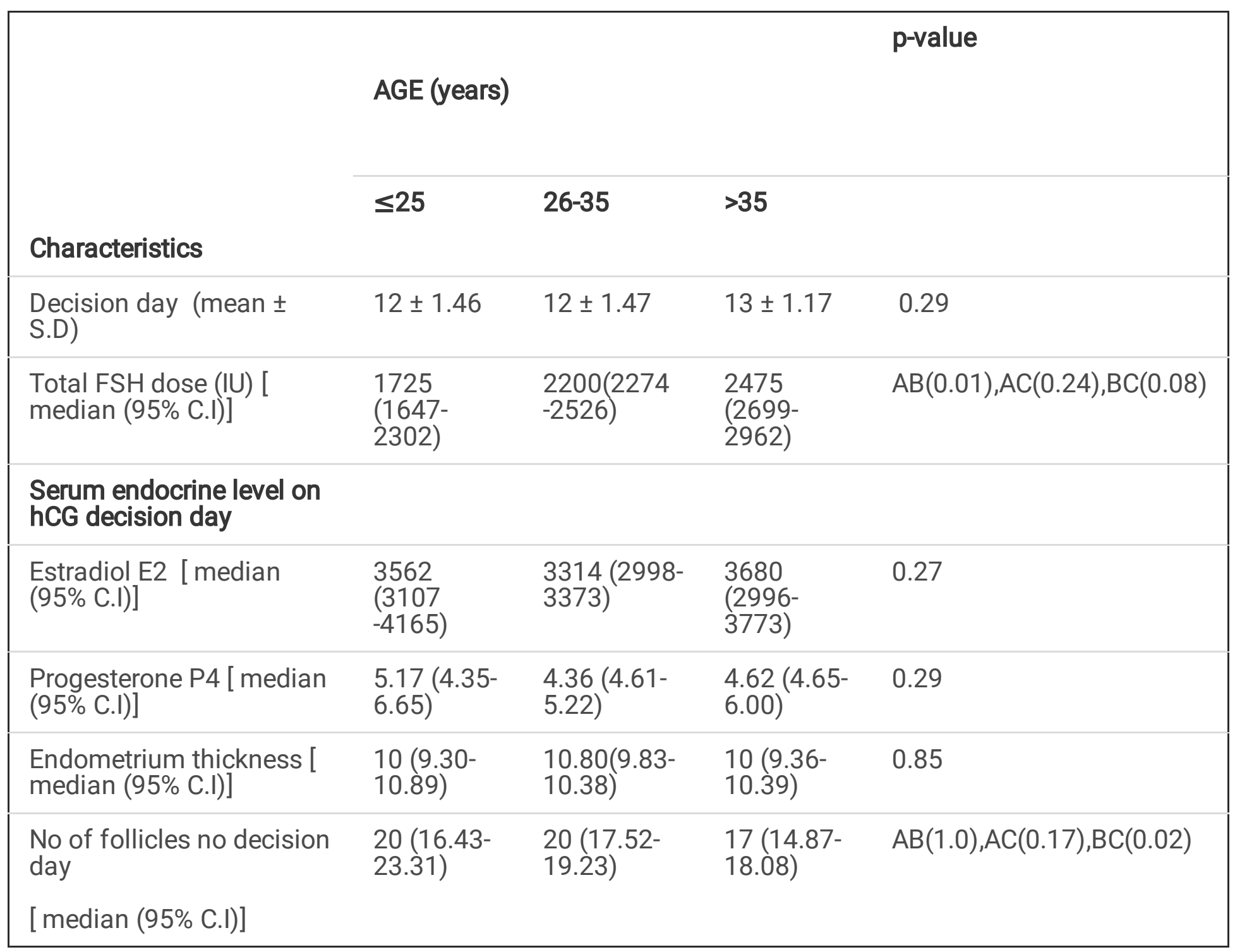

Table 2: stimulation parameters. GnRH dose (UI), Estradiol (E2), Progesterone (P4), Endometrium thickness, no of follicles and Decision day. For the kruskal wallis test 


\begin{tabular}{|lll|}
\hline Outcome parameters & Correlation coefficient $(\boldsymbol{r})$ & $\mathrm{p}$-value \\
\hline No. of follicles & -0.15 & 0.00 \\
\hline No. of eggs retrieved & -0.11 & 0.05 \\
\hline No. of embryo cleaved & -0.12 & 0.03 \\
\hline No. of fertilized embryos & -0.08 & 0.15 \\
\hline No. of transferred embryos & -0.09 & 0.11 \\
\hline
\end{tabular}

Table 3: Outcome parameters . No. of follicles, no. of eggs retrieved, no. of embryo cleaved, no. of fertilized embryos, no. of transferred embryos (Pearson correlation) *age

\begin{tabular}{|ll|}
\hline Chemical pregnancy & $\mathrm{N}(\%)$ \\
\hline Positive & $81(26.0)$ \\
\hline Negative & $206(66.2)$ \\
No ET & $24(7.7)$ \\
\hline
\end{tabular}

Table 4: Pregnancy Outcome

Figures 
ROC Curve

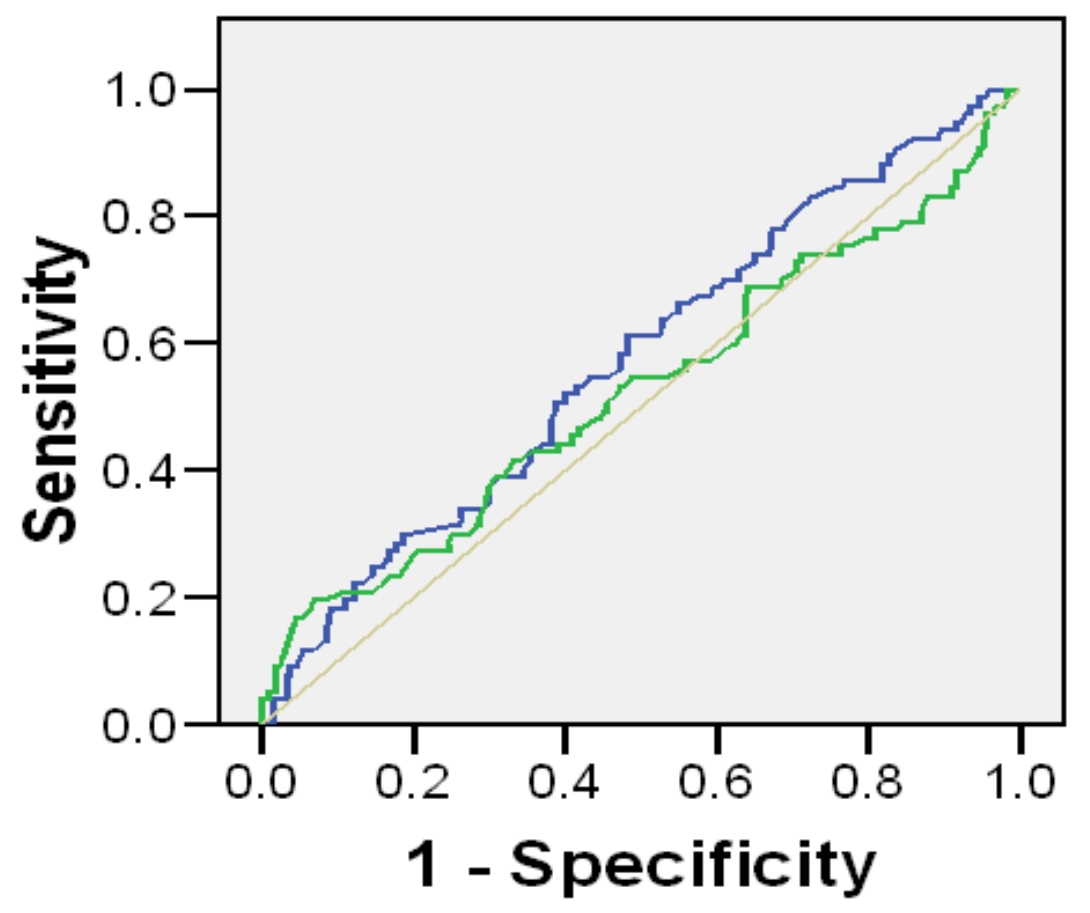

Source of the Curve

AMH.O

$\mathrm{FSH}$

Reference Line

$\mathrm{AMH}$ values

Area under the curve $=0.57$

Standard error $=0.03$

$\mathrm{p}$-value $=0.05$

C.I 95\% (lower-upper $)=(0.50-0.64)$

Cutoff value $=0.65$

Sensitivity $=1.00$

Specificity $=0.98$
FSH values

area under curve $=0.52$

Standard error $=0.04$

$\mathrm{p}$-value $=0.50$

C.I 95\% (lower-upper $)=(0.44-0.61)$

Cutoff value $=7.20$

Sensitivity $=0.54$

Specificity $=0.50$

Figure 1

ROC Curve 